\title{
CLAUDIO GROSSMAN: UN GENUINO COMPROMISO CON LA DEFENSA DE LOS DERECHOS HUMANOS.
}

*Laudatio leída el 3 de abril de 2019 por el decano de la Facultad de Derecho, profesor Alberto Balbontín Retamales, en la ceremonia de investidura como Doctor Honoris Causa por la Universidad de Valparaíso, del profesor Claudio Grossman.

La ceremonia de investidura de Doctor Honoris Causa constituye, a no dudarlo, el acto más solemne, y si se me permite, más elevado, en la vida de una institución universitaria. Se trata de la celebración de un rito de inusual ocurrencia, que no forma parte de la rutina de la universidad. Es, por el contrario, un acto excepcional, que solo en casos muy calificados se permite celebrar la institución, cuando quiere homenajear a quien, desde ese momento se convierte en parte de la comunidad que lo distingue y acoge, por el reconocimiento y admiración que le profesa, en mérito de la sobresaliente calidad del trabajo académico y profesional que ha desplegado durante su vida.

Es por lo mismo, que asumir el desafío de pronunciar las palabra que fundamentan esa distinción, pone al encargado en el trance de mostrar con entusiasmo y convicción, las virtudes y merecimientos del destinatario del homenaje, pero sin caer en la alabanza exagerada o frívola, ni dejarse llevar por los afectos personales, que muchas veces terminan por traicionar la naturaleza de un discurso que debe caracterizarse por su sobriedad académica. Con todo, no puedo dejar de manifestarme honrado, por la oportunidad de expresar estas palabras de presentación ante la comunidad de la Universidad de Valparaíso, referidas a un distinguido y admirado amigo de nuestra Facultad y, me atrevo a decir, de quien habla, que nació a la vida en esta ciudad, y al derecho en estas aulas.

En el caso de Claudio Grossman, hacer la presentación bajo las premisas de convicción y templanza, no ha sido fácil; pues reúne dos condiciones 
que dificultan el cumplimiento de la segunda de aquellas premisas, porque muestra un historial académico y profesional del máximo nivel y, a la vez, cuenta con una calidad, y una calidez humana, que no es fácil encontrar en personas que, como él, han alcanzado horizontes que para la mayoría quedan demasiado lejanos. Y, tengo que decirlo, probablemente han sido esas condiciones personales, las que le han facilitado el gran trabajo que ha desplegado desde sus inicios en el campo siempre complejo de la defensa de los Derechos Humanos. Casi, habría que decir, que la naturaleza lo dotó de esas virtudes humanas, como a un gran cantante pudo haberlo favorecido nacer con una voz privilegiada.

Dar cuenta de la obra de Claudio Grossman en un tiempo razonable, es una tarea compleja; porque su energía, su capacidad de trabajo, sus múltiples y diversos focos de interés, y su compromiso con aquellos temas que convocan su atención, se han traducido en decenas de actividades de diversa índole, que cubren tres ámbitos de su acción profesional, a saber, el académico, vertido en numerosos trabajos contenidos en libros de texto, destinados a la enseñanza del derecho internacional, y en artículos publicados en numerosas revistas especializadas. Un segundo ámbito, consistente en el ejercicio profesional, donde ha asumido la defensa de causas, tanto de Derechos Humanos, como de intereses nacionales; la más importante de estas últimas, sin dudas, fue la defensa de los intereses de nuestro país, en la singular demanda que entablara un país limítrofe en contra de Chile, y cuya defensa fuera brillante y exitosamente liderada por nuestro homenajeado, como Agente de Chile ante la Corte Interamericana de Justicia. Por último, en el campo de la docencia universitaria, donde creo yo, se puede hacer la diferencia con el primero de los ámbitos que hemos mencionado, donde se destaca en él su vivo interés por la formación de los nuevos abogados en el campo de la defensa y promoción de los Derechos Humanos. Con todo, no se pueden olvidar campos tan relevantes, como fue su muy extraordinaria gestión como Decano por más de 20 años, de la Facultad de Derecho de la American University en WDC, y que significó que se le nombrara Decano Emérito por dicha universidad, y se pusiera su nombre al aula mayor del nuevo edificio de la Facultad, inaugurado el año 2016, en señal de reconocimiento a su trayectoria, y a su trabajo como promotor fundamental de dicha magna obra. Tampoco puede olvidarse su permanente participación, cumpliendo roles destacados, en organizaciones profesionales y académicas de los EE. UU y extranjeras. 
Luego de obtener su grado de Doctor por la universidad de Amsterdam, con la tesis "El principio de no intervención en la organización de estados americanos", Claudio Grossman inició una muy fructífera carrera académica y profesional, enfocada en el Derecho Internacional, con particular dedicación a la defensa de los Derechos Humanos.

En el área más importante de destacar, por su aporte a la defensa de los Derechos Humanos, cabe mencionar su participación en calidad de abogado de las víctimas, en diversas causas llevadas ante el sistema americano de protección de tales derechos. Y probablemente, una de las más relevantes -destacada por el propio Grossman en diversas oportunidades- sea aquella caratulada Velásquez Rodríguez, Miguel Ángel con Honduras.

Una breve reseña del caso nos permitirá explicar algunos de los aspectos destacable de ese caso: Miguel Ángel Velásquez Rodríguez, era un estudiante universitario que se oponía a las políticas que en la época llevaba adelante el gobierno de su país. El 12.9.1981 fue detenido en Tegucigalpa. Según testigos, fue llevado junto a otros detenidos a un cuartel de las Fuerzas de Seguridad Pública, donde fue interrogado bajo torturas. 5 días después, el 17.9., se le trasladó a un recinto de las FFAA. Luego de eso, no se le volvió a ver. El gobierno negó su detención. El caso fue denunciado a la Com. Interamericana de Derechos Humanos el 7.10.81 la que, luego de infructuosas indagaciones y comunicaciones con el gobierno de Honduras, lo sometió al conocimiento de la Corte Interamericana de Derechos Humanos el 24.4.86. Con fecha 29.7.88, la Corte emitió su sentencia acogiendo la acusación, y declaró que el Estado de Honduras había violado los derechos de Miguel Ángel Velásquez Rodríguez.

1) La primera cuestión relevada, es de índole histórica: se trata del primer caso contencioso conocido por la Corte. Hay que recordar que la Corte se inauguró el 3.9.79, pero solo pudo conocer su primer caso contencioso, este, recién a partir de abril de 1986.

2) La segunda cuestión ya resulta de mayor interés dogmático. Se trata de que se acogió la tesis de que la desaparición forzada de personas, puede ser considerada un hecho atentatorio a la Convención ADH, e imputable a un estado, no obstante no encontrarse tipificada en el texto del tratado. La Corte lo expresa de la siguiente manera: "En la historia de la violación de los derechos humanos, las desapariciones no son una novedad. Pero su carácter sistemático y reiterado, su utilización como una técnica destinada a producir no sólo la desaparición misma, momentánea o permanente, de 
determinadas personas, sino también un estado generalizado de angustia, inseguridad y temor, ha sido relativamente reciente. Aunque esta práctica posee carácter más o menos universal, en América Latina ha presentado en los últimos años una excepcional intensidad. (149). El fenómeno de las desapariciones constituye una forma compleja de violación de los derechos humanos que debe ser comprendida y encarada de una manera integral.(150)". A partir de esa declaración, la Corte, agrega que "Si bien no existe ningún texto convencional en vigencia, aplicable a los Estados Partes en la Convención, que emplee esta calificación, la doctrina y la práctica internacionales han calificado muchas veces las desapariciones como un delito contra la humanidad". Agrega "El problema planteado exige [a la Corte] un examen sobre las condiciones en las cuales un determinado acto, que lesione alguno de los derechos reconocidos en la Convención, puede ser atribuido a un Estado Parte y comprometer, en consecuencia, su responsabilidad internacional.". En definitiva, de lo que se trata es de determinar si la violación a los derechos humanos resulta de la inobservancia por parte de un Estado de sus deberes de respetar y de garantizar dichos derechos, que le impone el artículo 1.1 de la Convención. [cuyo epigrafe es "Obligación de respetar los derechos"](173). Allí entonces, queda marcada la importancia que tiene la tesis sostenida por la defensa de las víctimas, al conseguir que se incorpore al estatuto de garantías de las personas, la proscripción de la desaparición forzada que hasta ese momento no se tipificaba, pero que luego se incorporó en el Estatuto de Roma, de la Corte Penal Internacional, como una de las modalidades del delito de lesa humanidad (art. 7 letra i),

3) Luego, resulta destacable la doctrina sentada por la Corte, relativa al estándar probatorio aplicable en estos casos, a partir de los argumentos esgrimidos por la defensa de las víctimas. En efecto, el Estado requerido, Honduras, sostuvo que la condena debía estar sustentada sobre hechos cuya prueba superara el estándar de la duda razonable. Como se sabe, dicho estándar se aplica en el derecho interno de muchos Estados para condenar a una persona, y exige un muy alto grado de convicción del juzgador. En este caso, los demandantes sostuvieron que, de aplicarse dicho criterio de valoración de la prueba, resultaría prácticamente imposible conseguir el reconocimiento de la violación al derecho de las víctimas, puesto que siendo un Estado el acusado, sus agentes, presuntamente comprometidos en los hechos, no iban a prestar ninguna colaboración a los investigadores de la Comisión. En tal virtud, el criterio debía ser que bastara con que las víctimas aportaran indicios, o pruebas circunstanciales, o medios de similar 
naturaleza, que dieran verosimilitud a la acusación, trasladándose el peso de la prueba al Estado, a fin de que este probara que había cumplido con su compromiso de proteger los derechos de los individuos, conforme lo exige el artículo 1.1 de la Convención. Razona la Corte en los siguientes términos: "En efecto, la protección internacional de los derechos humanos no debe confundirse con la justicia penal. Los Estados no comparecen ante la Corte como sujetos de acción penal. El Derecho internacional de los derechos humanos no tiene por objeto imponer penas a las personas culpables de sus violaciones, sino amparar a las víctimas y disponer la reparación de los daños que les hayan sido causados por los Estados responsables de tales acciones.(134) A diferencia del Derecho penal interno, en los procesos sobre violaciones de derechos humanos, la defensa del Estado no puede descansar sobre la imposibilidad del demandante de allegar pruebas que, en muchos casos, no pueden obtenerse sin la cooperación del Estado.(135). Es el Estado quien tiene el control de los medios para aclarar hechos ocurridos dentro de su territorio. La Comisión, aunque tiene facultades para realizar investigaciones, en la práctica depende, para poder efectuarlas dentro de la jurisdicción del Estado, de la cooperación $y$ de los medios que le proporcione el Gobierno"(136). Con base en ese criterio, da por establecidos los hechos fundantes de la acusación.

4) Por último, también resulta destacable la autorización de la Comisión, para que se incorporaran los abogados de las víctimas al equipo de la acusación. Como lo explica Claudio Grossman en un artículo denominado "Desapariciones en Honduras: La necesidad de una representación directa de las víctimas en los litigios de DDHH", publicado el año 1992, el problema se generaba a partir del talante tradicional del DI, cuyos sujetos son los Estados u organismos especialmente autorizados para actuar en el ámbito de la comunidad internacional, pero no las personas. Solo después de la II Guerra Mundial, con la tragedia del Holocausto y demás sistemas de exterminio a ciertas minorías, el Derecho Internacional comenzó a reconocer derechos individuales. Sin embargo, en el ámbito procesal, esto es, para su ejercicio, se mantienen las restricciones para que las personas puedan intervenir directamente en los litigios, lo que se convierte en una importante limitación al ejercicio de sus derechos, pues, cuando la representación la asumen los Estados, las víctimas no tienen certeza de que éstos vayan a comprometerse con sus demandas, puesto que actúan motivados por intereses políticos, económicos o de seguridad, y no por consideraciones propiamente humanitarias. De allí es que, sin perjuicio de plantear en dicho trabajo la necesidad de que por la naturaleza de los hechos 
que se siguen en el marco de las causas por Interamericana de Derechos Humanos, las personas individuales deban tener la posibilidad intervenir directamente a través de abogados de su confianza, en el caso Velázquez vs. Honduras, el problema se resolvió consiguiendo que la Comisión les nombrara como asesores. Eso les permitió participar en la planificación de la estrategia, lo que, a su vez, sirvió para que el litigio se condujera por la vía que les interesaba a las víctimas, esto es, concentrándose en los hechos, y no en los aspectos formales como pretendía el Estado denunciado.

Pero ya decía al inicio, Claudio Grossman ha tenido una participación destacada en la defensa de los Interamericana de Derechos Humanos, al punto que ha pasado de asumir la defensa directa de las víctimas, a constituirse en un líder encargado de conducir organismos internacionales, tales como la Comisión Interamericana de Interamericana de Derechos Humanos, cuya presidencia ostentó los años 1996-1997 y 2001, o la del Comité contra la Tortura, dependiente de las Naciones Unidas. En el primer caso no solo se ocupó de las denuncias que recibió la Comisión durante su presidencia, sino que normalizó algunos procesos que no se había podido regular debidamente, dadas las graves condiciones políticas que afectaban a buena parte del continente, varios de cuyos países padecían regímenes dictatoriales, con graves y masivas violaciones a los Interamericana de Derechos Humanos.

Entre los múltiples temas que ha abordado Claudio Grossman en su atención por los Interamericana de Derechos Humanos, hay uno que suele aparecer con frecuencia en sus trabajos. Me refiero a la libertad de expresión. Su interés por ese derecho queda de manifiesto en sus escritos, a partir de su convicción de que se trata, ni más ni menos, que de un derecho o una libertad, que es de la esencia de cualquier sistema democrático digno de ese nombre. Para Claudio Grossman la libertad de expresión "es un contrapeso fundamental que permite obtener y difundir opiniones e información, fortaleciendo la sociedad civil y creando posibilidades de participación para los individuos" (Nos ad justitiam... p 1031). Para él la libertad de expresión abarca no solo la libertad de emitir opiniones, sino la de recibir toda la información que se requiera por los ciudadanos sobre los asuntos de interés público. A su juicio, las leyes que establecen delitos de injuria y calumnia, debieran derogarse y reemplazarse por un estatuto de responsabilidades civiles, cuando se incurre en excesos. Sin embargo -a partir de su experiencia práctica- advierte que cuando las consecuencias civiles son demasiado gravosas, terminan operando como verdaderos 
disuasivos al ejercicio de la libertad de expresión. También se manifiesta contrario a cualquier forma de monopolio, público o privado, del manejo de la información. Postula, por otra parte, el deber de los estados de garantizar efectivamente el acceso a la información que se encuentre en poder de organismos sea públicos o privados. En esa línea, plantea la necesidad de que se entregue una formación especializada en la materia a jueces y abogados, a partir del marco normativo y jurisprudencial que rige en el Derecho Internacional, de modo que se facilite la prevención y solución interna de los conflictos, reduciendo la necesidad de recurrir a instancias internacionales.

Los años 2008 y 2015 ejerció el cargo de presidente del Comité de las Naciones Unidas contra la Tortura, donde, entre otras materias, se ocupó especialmente del problema de la reparación a las víctimas; cuestión que reviste especial complejidad, ya que excede la mera indemnización o la atención de salud básica y de urgencia, sino que abarca aspectos más sofisticados, como la rehabilitación física y síquica, y el compromiso de los Estados de atender sus necesidades, sin importar dónde ni de quién sufrió la tortura. El año 2009, presentó un borrador a la Comisión, proponiendo elaborar una Observación General sobre el artículo 14 del Convenio CT, lo que derivó en la formación de un grupo de trabajo dentro de la misma, así como la presentación de un texto alternativo por otros comisionados, y la invitación a los Estados y ONG, para que hicieran sus aportes, de modo que hubiere criterios compartidos sobre este sensible tema.

El año 1998, en su condición de Relator Especial Sobre Los Derechos De La Mujer, designado por la CIDH, presentó el "Informe sobre la Condición de la Mujer en las Américas" donde expresa: "El contenido de este informe demuestra que hay discriminación de jure de la mujer en varios países, especialmente con relación a asuntos familiares, administración de bienes y el sistema penal. En los casos de ausencia de discriminación de jure, la práctica actual muestra que importantes derechos de la mujer están desprotegidos. Esta situación afecta en casi todos los ámbitos sociales y a prácticamente todos los países de la región. La pobreza y los conflictos armados tienen un efecto negativo y desproporcionado sobre las mujeres. Además, las mujeres miembros de grupos indigenas o de minorías étnicas están expuestas a otras serias violaciones que son el resultado de su situación especifica".

Pero debo destinar al menos un párrafo a comentar lo que ha sido uno de los ámbitos más vitales de su preocupación por los Interamericana de 
Derechos Humanos: Me refiero a la formación jurídica en dicha materia. Claudio Grossman ha desplegado una incansable atención tanto en la formación de los futuros abogados, como también en la de quienes han tenido el privilegio de llegar a su Facultad a cursar programas de postgrado. En todos los casos la impronta de Claudio Grossman ha sido la relevancia de los Interamericana de Derechos Humanos en el trabajo de los abogados. Y a partir de allí, también lo ha sido la insistencia de que ya no es posible considerar que el Derecho Internacional es una cuestión de Estados, sino que los individuos se encuentran cada vez más involucrados en situaciones que los afectan directamente desde diversos estatutos jurídicos nacionales, siendo necesario, por tanto, poner mayor atención a los fenómenos jurídicos que tienen su base en el Derecho Internacional. En un artículo que titula "Educación jurídica global y derechos humanos", afirma: "Las escuelas de derecho desempeñan un papel vital en el establecimiento de expectativas legítimas de comportamiento en las sociedades. Por lo tanto, la educación en derechos humanos es crucial para la promoción de importantes valores. Hoy se está produciendo una transformación dramática en el mundo. Los procesos que han llevado a esta situación han dado lugar a un aumento del comercio y la inversión transnacionales, crecimiento espectacular en el alcance y la velocidad de las comunicaciones en todo el mundo, y una exposición sin precedentes a las culturas y valores extranjeros. Internet, con su capacidad de trascender fronteras y permitir una comunicación descentralizada, es tanto una metáfora como un potente motor que contribuye cada vez más a un mundo sin barreras. Han surgido nuevos desafios en materia de seguridad, medio ambiente, salud, protección laboral, y gobernanza. Los estados nacionales ya no son los actores más importantes en abordar eficazmente muchos de estos desafios." Agrega: "Una realidad más compleja ha sustituido la clara dicotomía entre lo doméstico y las cuestiones internacionales, y la distinción entre lo nacional y lo internacional está perdiendo su significado. Esta situación afecta a todos los estados en diferentes grados. Algunos estados pueden soportar mayor peso político, económico, militar o ideológico para enfrentar los nuevos desafios, pero ningún estado puede ignorarlos. El impacto de esta nueva realidad mundial, y su efecto en el ámbito de la jurisdicción doméstica, ha dado lugar a graves conflictos y debates sobre sus beneficios para sociedades e individuos". En esa línea entonces, es que postula la necesidad de que los estudiantes de derecho, y sus profesores, asuman la realidad de que ya no basta con conocer derecho civil, procesal, penal o comercial de cada uno de sus países, sino que deben considerar los 
elementos normativos que proporciona el Derecho Internacional. Razona a partir de la experiencia de las dos guerras mundiales que sufrió la humanidad el siglo pasado, con la desastrosa destrucción que significaron por si mismas, pero agregando las gravísimas violaciones a los derechos de millones de seres humanos, víctimas del genocidio nazi, y también el posterior desarrollo de armas de destrucción masiva. Todo ello demuestra, a su juicio, que ya no basta con que los Estados invoquen el principio de soberanía absoluta; porque no es suficiente para garantizar el ejercicio pleno de los derechos de los individuos y "el bienestar y supervivencia de la humanidad". Es esencial, dice, que los estudiantes aprendan a enfrentar los casos tomando en consideración diversas tradiciones jurídicas. Darles esa formación, favorece, dice, la tolerancia "un valor crucial en la protección de los DDHH". En su opinión, "Las escuelas de derecho deben reflejar la nueva diversidad, para que los graduados puedan interactuar y estar preparados para trabajar en un mundo que es multinacional y pluralista.". La globalización, ha traído muchos beneficios, pero también grandes problemas y desafíos que afectan a toda la humanidad, y que dan lugar a que se generen fenómenos de violencia y terrorismo, que encuentran justificación en dramas que son reales, pero que significan la negación de principios básicos de los Interamericana de Derechos Humanos y de la civilización. De allí es que los abogados deben cumplir un rol muy importante en el manejo de los conflictos, y en la definición de expectativas legítimas de comportamiento, y promover el valor de la dignidad humana.

En fin, es tanto lo que se puede decir sobre el pensamiento y la acción de Claudio Grossman, que continuar sería atentar contra el derecho de todos ustedes a agotarse escuchándome. Por eso dejaré hasta acá las referencias a la obra de nuestro invitado, no sin antes declarar que solo he comentado una mínima fracción de su trabajo, y confesar, además, que elegí los temas algo al azar. Porque es tanto y tan diverso su despliegue de energía, que hace imposible sintetizar todo en unos breves minutos.

Quiero terminar diciendo que el rol que ha jugado nuestro homenajeado en los diversos campos vinculados a la defensa de los Interamericana de Derechos Humanos, tanto desde la cátedra, como desde los más altos cargos de organismos internacionales, y en el ejercicio mismo de la abogacía, me lleva a compartir con ustedes una reflexión que deriva de su propia experiencia de vida, de la que por respeto a Claudio no daré mayores detalles. Pero debo decir que salió de Chile hacia Holanda en 1974, en calidad de asilado. Y sufrió el exilio durante 14 años. Sin embargo, ese 
desgarro personal y familiar, no fue motivo para que cultivara odio ni resentimiento contra los que no piensan como él, o infligieron daño a él y a su familia. Muy por el contrario, aplicó toda su inteligencia, su energía y su sobresaliente capacidad de trabajo, para ir literalmente por el mundo, promoviendo la tolerancia y la defensa y promoción de los Interamericana de Derechos Humanos, sin importar quienes demandaran su atención. Francamente emociona comprobar tan notable ejemplo de nobleza, integridad moral y coherencia jurídica (los derechos en serio, diría Dworkin) de quien no mira el color político de la víctima, o del victimario, y luego actúa o se omite en su compromiso con la causa que proclama. Es por ello por lo que me atrevo a señalarlo como un ejemplo de lo que debe ser un auténtico defensor de la noble causa de los Interamericana de Derechos Humanos.

En nombre de tantos hombres y mujeres de América y el mundo que se han beneficiado de su infatigable trabajo, de nuestra Universidad de Valparaíso, y en estas aulas que un día le acogieron en la adolescencia, le digo: muchas gracias profesor Claudio Grossman. 